\title{
Acompanhamento da evolução dos distúrbios de imagem corporal em pacientes com bulimia nervosa, ao longo do tratamento multiprofissional
}

\author{
Longitudinal assessment of body image disturbances in patients with bulimia nervosa submitted to \\ multidisciplinary treatment
}

\author{
Fernanda Timerman ${ }^{1}$, Fernanda Baeza Scagliusi², Táki AthanÁssios Cordás 3 \\ 1 Máster em Educação por pesquisa da Universidade de Sydney, Austrália. \\ 2 Doutora em Educação Física pela Universidade de São Paulo (USP), com pós-doutorado em Nutrição em Saúde Pública pela USP. Professora adjunta da Universidade Federal de São Paulo \\ (Unifesp), Campus Baixada Santista, Departamento de Ciências da Saúde. \\ ${ }^{3}$ Coordenador-geral do Ambulatório de Bulimia e Transtornos Alimentares (Ambulim) do Instituto de Psiquiatria do Hospital das Clínicas da Faculdade de Medicina da Universidade de São Paulo \\ (IPq-HCFMUSP).
}

Recebido: 20/7/2009 - Aceito: 22/10/2009

\section{RESUMO}

Objetivo: Aumentar a compreensão sobre os distúrbios da imagem corporal em pacientes com bulimia nervosa e sua evolução após o tratamento multidisciplinar. Métodos: Onze mulheres responderam dois questionários de imagem corporal (Body Attitudes Questionnaire e Escala de Figuras de Stunkard) antes e após o tratamento e preencheram o diário alimentar, utilizado para a análise de sintomas de bulimia nervosa. Resultados: Tanto no pré quanto no pós-tratamento o índice de massa corporal médio se encontrava na faixa de eutrofia. Cerca de 57,0\% da amostra teve remissão total dos sintomas de bulimia nervosa. Em relação à imagem corporal, na escala de Stunkard, o tamanho da figura escolhida como ideal aumentou e houve redução da insatisfação corporal após o tratamento. Não houve redução significativa da superestimativa do tamanho corporal, porém no início do tratamento $57,1 \%$ das pacientes já não superestimavam seu tamanho. Em relação ao questionário de atitudes, notou-se diminuição das seguintes variáveis: depreciação corporal; sentir-se gorda; sensação de ter gordura nos membros inferiores e importância pessoal do corpo. Conclusões: $\mathrm{O}$ tratamento foi eficaz em alguns aspectos atitudinais, porém deve ser aprimorado em outros, como a distorção perceptual.

Timermam F, et al. / Rev Psiq Clín. 2010;37(3):113-7

Palavras-chave: Bulimia nervosa, imagem corporal, tratamento multiprofissional.

\begin{abstract}
Objective: Increase the comprehension of body image disturbances in patients with bulimia nervosa and its evolution after treatment, multidisciplinary. Methods: Eleven women answered two body image questionnaires (Body Attitudes Questionnaire and Stunkard's Figure Scale) before and after treatment and filled a food diary used to analyze the frequency of bulimia nervosa symptoms. Results: The mean body mass index was found at normal weight category before and after treatment. Approximately $57.0 \%$ of the sample had total remission of bulimia nervosa symptoms. In relation to body image based on Stunkard's scale, the size of the figure chosen as ideal increased and body dissatisfaction decreased after treatment. There was no significant reduction in the overestimation of body size, but at the beginning of the treatment $57,1 \%$ of the patients did not overestimate their sizes already. Regarding the questionnaire of attitudes, the following variables had a significant decrease: body depreciation, feeling fat, sensation of having lower body fatness and body self-importance. Discussion: The treatment was effective in some attitudinal aspects, but should be improved in regard to others, such as perceptual distortion.
\end{abstract}

Timermam F, et al. / Rev Psiq Clín. 2010;37(3):113-7

Keywords: Bulimia nervosa, body image, multidisciplinary treatment.

\section{Introdução}

Os critérios diagnósticos da bulimia nervosa (BN) estão focados nos comportamentos de compulsão alimentar com subsequente purgação, bem como autoavaliação indevidamente influenciada pela forma corporal e peso. Estudos ${ }^{1-3}$ apontam a imagem corporal como um componente crítico dos transtornos alimentares (TA), visto que a disfunção dessa imagem pode levar a comportamentos extremos para atingir um ideal de beleza muitas vezes inatingível. $\mathrm{Na}$ $\mathrm{BN}$, a tendência é de insatisfação decorrente da avaliação negativa da imagem corporal 4 .

A imagem corporal é definida como "a figura que temos em mente do tamanho e forma dos nossos corpos e os nossos sentimentos em relação a essas características e partes constituintes do corpo" 4 . A imagem corporal possui dois componentes principais: atitudinal e perceptual 4 .
O componente atitudinal pode ser didaticamente dividido em três aspectos: a) pensamentos e crenças que um indivíduo tem sobre sua aparência (denominado componente avaliativo); b) experiências corporais emocionais (componente chamado de afeto) e c) importância da aparência para o indivíduo e comportamentos adotados para mantê-la ou melhorá-la (componente denominado de investimento $)^{5}$. O componente perceptual corresponde à forma pela qual o indivíduo vê ou percebe seu corpo. Assim, um indivíduo que sub ou superestima seu tamanho corporal possui uma distorção perceptual6. Resumidamente, a noção da imagem corporal é primeiramente um fenômeno perceptual, porém o julgamento que o indivíduo faz daquilo que ele percebe deriva de fatores cognitivos, atitudinais e afetivos.

Uma metanálise ${ }^{7}$ obteve a informação de que os pacientes com TA possuem distúrbios atitudinais de imagem corporal, nos quais não havia superestimativa do tamanho corporal e sim profunda insa-

Instituição onde o trabalho foi realizado: Ambulatório de Bulimia e Transtornos Alimentares (Ambulim) do Instituto de Psiquiatria do Hospital das Clínicas da Faculdade de Medicina da Universidade de São Paulo (IPq-HCFMUSP).

Endereço para correspondência: Fernanda Timerman. Rua Dr. Ovídeo Pires de Campos, 785, Ambulim - 05403-010 - São Paulo, SP. Telefone: (11) 3069-6975. E-mail: fe.timerman@gmail.com 
tisfação com seus corpos. Segundo Skrzypek et al. ${ }^{1}$, as pacientes com TA que superestimam seu tamanho corporal devem ser um subgrupo específico, que, inclusive, apresentaria pior prognóstico.

Existem controvérsias que precisam ser mais amplamente estudadas a respeito das disfunções perceptuais e atitudinais para analisar como cada uma incide em pacientes com TA.

Uma limitação da maioria dos estudos é a redução do componente atitudinal da imagem corporal à insatisfação e preocupação com o corpo, o que certamente reduz as possibilidades de exploração acerca da natureza das disfunções da imagem corporal ${ }^{5}$. Alguns estudos foram além, utilizando o Body Attitudes Questionnaire (BAQ) ${ }^{8}$, que mede mais profundamente o grau de distúrbios atitudinais.

A eficácia da intervenção multiprofissional oferecida pelo Ambulim nos distúrbios de imagem corporal em pacientes com BN também já foi examinada, tanto por Alvarenga ${ }^{9}$ quanto por Cordás ${ }^{10}$. Ambos relatam redução dos escores de distúrbios da imagem corporal com base no Body Shape Questionnaire (BSQ) em pacientes com $\mathrm{BN}$. Entre todos os componentes da imagem corporal que podem estar prejudicados na BN, ele só avalia preocupação com a forma e o tamanho corporal.

O objetivo desse estudo foi acompanhar a evolução dos distúrbios da imagem corporal em pacientes com $\mathrm{BN}$, ao longo do tratamento multiprofissional, oferecido pelo Ambulatório de Bulimia e Transtornos Alimentares (Ambulim), utilizando outros instrumentos que medem os componentes perceptuais e atitudinais da imagem corporal. Como forma de controle, os resultados foram comparados com aqueles descritos na literatura acerca dos efeitos de diversos tratamentos sobre os distúrbios de imagem corporal em pacientes com TA.

\section{Métodos}

\section{Local de estudo}

O estudo foi realizado no Programa de Transtornos Alimentares do Ambulim do Instituto de Psiquiatria do Hospital das Clínicas da Faculdade de Medicina da Universidade de São Paulo (HCFMUSP). O estudo foi aprovado pelo Comitê de Ética do Hospital das Clínicas e as voluntárias assinaram um termo de consentimento livre e esclarecido antes de participar.

\section{Amostra}

Foram contatadas aproximadamente 100 mulheres cadastradas na lista de espera para uma triagem inicial por telefone. Alguns dados como idade, alfabetização, disponibilidade de tempo, estar sob algum tratamento psiquiátrico, psicológico ou nutricional ou em uso de algum medicamento foram verificados. Das 100 mulheres, apenas 30 eram elegíveis e foram convocadas para triagem clínica na unidade. Dessas, somente 14 não preenchiam nenhum critério de exclusão e preenchiam tanto o critério diagnóstico de $\mathrm{BN}$ como também os critérios de inclusão para participarem do tratamento.

Essas 14 mulheres atenderam aos seguintes critérios de inclusão: preencherem diagnóstico para $\mathrm{BN}$ segundo os critérios do DSM-IVTR 11 , terem disponibilidade para as dezoito semanas de tratamento; serem alfabetizadas e capazes de entender os instrumentos de avaliação a serem respondidos; serem maiores de 18 anos. Os critérios de exclusão eram: estarem em tratamento psiquiátrico, psicológico ou nutricional há mais de três meses antes do início do tratamento no serviço, possuírem índice de massa corporal (IMC) inferior a 18,5 ou acima de $34,9 \mathrm{~kg} / \mathrm{m}^{2}$; presença de quadro psicótico ou depressão grave com pontuação $\geq 25$ na escala de Hamilton para Depressão ${ }^{12}$, e estarem em uso de medicação psiquiátrica. Após o início do tratamento, o uso de psicofármacos foi prescrito no caso de o tratamento psicoterápico nao ter evoluído como o esperado. Nesse caso, os antidepressivos, principalmente os tricíclicos e os inibidores seletivos de recaptura da serotonina (ISRS), juntamente com a administração de anticonvulsivante (Topiramato), foram mais amplamente utilizados para redução da frequência de episódios de BN e vômitos, além de atuar em sintomas ansiosos e depressivos, quando presentes. A dosagem variou de acordo com a necessidade individual ${ }^{13}$.

Em relação às características gerais da amostra, a maior parte $(57,1 \%)$ cursou até o ensino superior completo e era solteira $(71,2 \%)$. Em relação à etnia, a maioria da amostra era branca $(64,3 \%)$, seguida de oriental $(28,6 \%)$ e negra $(7,1 \%)$. O tempo em que as participantes tinham a doença oscilou bastante, sendo o mínimo de 1 ano e o máximo de 21 anos. A maioria das participantes (6) relatou 1 a 5 anos de $\mathrm{BN}$; quatro relataram entre 6 e 10 anos; uma relatou entre 11 e 15 anos e três delas relataram ter $\mathrm{BN}$ há mais tempo (de 16 a 21 anos). Ao longo do tratamento, três $(21,4 \%)$ das 14 participantes da amostra inicial desistiram e foram então descartadas das análises seguintes. A amostra final foi composta por 11 mulheres.

\section{Desenho do estudo}

Após o processo de recrutamento e inclusão das pacientes no tratamento, as 14 remanescentes preencheram o termo de consentimento livre e esclarecido e dois questionários para avaliação da imagem corporal e também foram instruídas a preencher os diários alimentares de sete dias.

Essas pacientes receberam tratamento multiprofissional como descrito adiante. Ao término da intervenção, os mesmos instrumentos iniciais foram reaplicados. O peso corporal foi medido antes e depois do tratamento em balança convencional. A altura foi relatada pelas próprias pacientes, pois o Ambulatório não dispunha de estadiômetro para tal mensuração. Os diários alimentares foram utilizados para análise pré e pós-tratamento da frequência de vômitos, compulsões, uso de diuréticos, laxantes e medicações para perda de peso e horas de prática de exercício físico.

\section{Avaliação psiquiátrica}

Todas as pacientes foram entrevistadas por um psiquiatra júnior e um sênior por meio de uma anamnese clínica semiestruturada, com o objetivo de diagnosticar a presença de $\mathrm{BN}$ e outras comorbidades segundo os critérios do DSM-IV, pois é o manual mais usado na literatura internacional de TA, aumentando a possibilidade de comparar os dados do presente estudo com tais dados da literatura, além de coletar dados clínicos para outros braços do mesmo estudo.

\section{Avaliação da imagem corporal}

A avaliação da imagem corporal foi baseada em dois instrumentos que abordam os componentes perceptuais e atitudinais do construto imagem corporal.

$\mathrm{O} B A Q^{8}$ é um questionário autoaplicável de 44 itens que foi desenvolvido com base nas preocupações com o corpo de uma larga amostra de mulheres australianas e visa mensurar diversas atitudes em relação ao corpo. As respostas são agrupadas em seis subescalas, que, por sua vez, descrevem: (1) sentimentos a respeito da adiposidade corporal em geral (denominada "Sentir-se gorda"); (2) sentimentos de aversão e repulsa relacionados ao corpo (denominada "Depreciação"); (3) sentimentos de força e aptidão física individual (chamada de "Força e aptidão física"); (4) relevância pessoal do peso e da forma corporais (nomeada de "Saliência"); (5) achar-se fisicamente atraente, primariamente em relação ao sexo oposto (denominada "Atração física"); (6) sentimentos de que os membros inferiores do corpo são gordos (chamada de "Gordura nos membros inferiores"). Altos escores nas subescalas "Sentir-se gorda", "Depreciação", "Saliência" e "Gordura nos membros inferiores" implicam atitudes negativas em relação ao corpo. Altos escores nos quesitos "Força e aptidão física" e "Atração física" indicam atitudes corporais positivas. Na tradução para a língua portuguesa, ele se mostrou um instrumento de boa validade convergente e discriminatória, além de preciso (os coeficientes de correlação teste-reteste variaram entre 0,57 e 0,85$)^{14}$.

A escala de figuras de Stunkard ${ }^{15}$ consiste em uma escala de nove figuras femininas, variando de uma figura muito magra, represen- 
tada pelo número 1, até uma figura muito obesa, representada pelo número 9. As pacientes deveriam escolher, entre essas nove figuras, uma que representasse seu tamanho corpóreo atual (identificando como EU); uma que representasse, na opinião delas, a figura saudável (identificando como SAUDÁVEL) e a figura que elas desejariam ser (identificando como IDEAL). Essa escala produz cinco variáveis: 1 ) número correspondente à figura atual; 2) número correspondente à figura saudável; 3) número correspondente à figura ideal; 4) escore da discrepância entre as figuras saudável e atual (subtrai-se o número correspondente à figura saudável do número correspondente à atual); e 5) escore da discrepância entre as figuras ideal e atual (subtrai-se o número correspondente à figura ideal do número correspondente à atual). Esse questionário foi traduzido e validado em português por Scagliusi et al. ${ }^{16}$. O escore de discrepância entre as figuras saudável e atual é uma medida de insatisfação corporal mais realista e razoável, enquanto o escore de discrepância entre as figuras ideal e atual reflete uma decisão mais emocional ${ }^{16}$.

Com base em uma larga amostra de mulheres caucasianas, Bulik et al. ${ }^{17}$ determinaram que as silhuetas 1,2 e 3 correspondem ao IMC menor que $20 \mathrm{~kg} / \mathrm{m}^{2} \mathrm{e}$ as silhuetas maiores ou iguais a 6 correspondem ao IMC maior ou igual a $30 \mathrm{~kg} / \mathrm{m}^{2}$. Tanto mulheres com IMC menor que $20 \mathrm{~kg} / \mathrm{m}^{2}$ que escolhem silhuetas maiores ou iguais a 4 como representativa da silhueta atual quanto mulheres com IMC menor que $30 \mathrm{~kg} / \mathrm{m}^{2}$ que escolherem silhuetas maiores ou iguais a 6 estão superestimando seus tamanhos corporais. Assim, é possível determinar a proporção de mulheres que superestimam seus tamanhos corporais, conforme foi feito no estudo de Scagliusi et al. ${ }^{16}$.

\section{Tratamento}

O tratamento foi realizado por equipe multiprofissional e ocorreu uma vez por semana, durante 18 semanas. Semanalmente, as pacientes participavam das seguintes atividades: consulta individual com o psiquiatra, grupo de educação nutricional, consulta individual com o nutricionista e psicoterapia em grupo com enfoque cognitivocomportamental. Durante o período do Ambulatório, as pacientes tinham de participar integralmente de todas as atividades. Caso a paciente tivesse mais de três faltas sem justificativa plausível, seria dada alta por abandono.

\section{Análise estatística}

As análises foram conduzidas com o auxílio do software SPSS (Statistical Package for Social Sciences, versão 10.0, 1999). As variáveis analisadas foram: 1) escores das seis subescalas do BAQ; 2) número correspondente à figura atual; 3 ) número correspondente à figura saudável; 4) número correspondente à figura ideal; 5) escore da discrepância entre as figuras saudável e atual; 6) escore da discrepância entre as figuras ideal e atual; 7) peso corporal, 8) frequência de compulsões; e 9) frequência de purgações totais.

Chamou-se de frequência de purgações totais a soma das frequências semanais de vômitos autoinduzidos, uso de remédios antiobesidade, uso de laxantes e de diuréticos. Não foi incluída a prática de atividade física por apresentar unidade diferente das outras variáveis.

Foram utilizados o teste de Shapiro-Wilk para verificar se as variáveis possuíam distribuição normal e o teste de Levene para verificar se as variáveis possuíam variâncias homogêneas. Tais condições não foram cumpridas e, por isso, optou-se por testes não paramétricos, tais como: teste de Wilcoxon para comparação de variáveis no pré e no pós-tratamento; e o teste do Qui-quadrado para analisar a proporção de pacientes que superestimavam seu tamanho corporal nos dois momentos. Para comparar os distúrbios de imagem corporal entre as pacientes com e sem remissão de sintomas, foi utilizado o teste de Mann-Whitney. Chamou-se de remissão de sintomas a isenção de quaisquer sintomas de BN, ou seja, episódios de compulsão alimentar seguidos de purgação.

\section{Resultados}

Das 14 participantes iniciais, $12(85,7 \%)$ possuíam peso normal, uma $(7,1 \%)$ tinha sobrepeso e uma $(7,1 \%)$ era obesa. Não houve diferença significativa de IMC no pós-tratamento, porém houve tendência para um pequeno aumento $(\mathrm{p}=0,6)$, tendo em vista que esse índice, no pré-tratamento, foi de $22,7 \pm 4,1$ e, no pós-tratamento, de 23,0 $\pm 4,5$. Tanto no pré quanto no pós-tratamento o IMC médio se encontrava na faixa de eutrofia $\left(18,5 \leq \mathrm{IMC}<25 \mathrm{~kg} / \mathrm{m}^{2}\right)$. Das 11 $(78,6 \%)$ pacientes que permaneceram, $8(57,2 \%)$ tiveram remissão total dos sintomas.

A figura 1 mostra as frequências semanais de compulsões e purgações totais nos períodos pré e pós-tratamento.

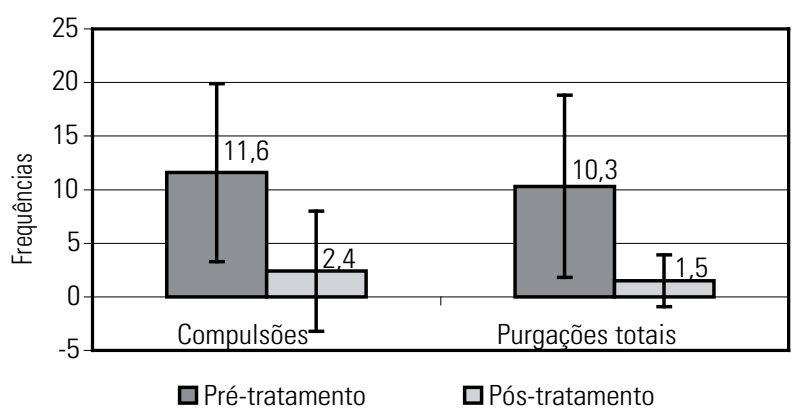

Figura 1. Frequências semanais de compulsões e purgações totais nos períodos pré e pós-tratamento (média \pm desvio-padrão). Compulsões $(z=-2,25, p=0,02)$; purgações $(Z=-2,81, p=0,005)$.

Houve diminuição significativa no pós-tratamento tanto das compulsões $(z=-2,25, p=0,02)$ quanto das purgações $(Z=-2,81$, $\mathrm{p}=0,005)$.

A tabela 1 discorre os resultados da escala de figuras de Stunkard.

Tabela 1. Distribuição das variáveis de imagem corporal (em média, desviopadrão, mediana, mínimo e máximo) da escala de figuras de Stunkard, nos períodos pré e pós-tratamento

\begin{tabular}{l|c|c}
\hline & Pré-tratamento & Pós-tratamento \\
\hline Número da figura escolhida como atual & $5,0 \pm 1,8(5 ; 2-8)$ & $3,9 \pm 1,5(4 ; 4-9)$ \\
\hline $\begin{array}{l}\text { Número da figura escolhida como } \\
\text { saudável }\end{array}$ & $2,6 \pm 0,9(2 ; 1-4)$ & $3,3 \pm 0,7(3 ; 2-4)$ \\
\hline Número da figura escolhida como ideal & $1,5 \pm 0,7(1 ; 1-3)$ & $2,4 \pm 0,7(2 ; 2-4)^{*}$ \\
\hline $\begin{array}{l}\text { Discrepância entre a figura atual e a } \\
\text { saudável }\end{array}$ & $2,4 \pm 2(3 ; 0-6)$ & $1,6 \pm 1,7(1 ; 0-6)^{*}$ \\
\hline $\begin{array}{l}\text { Discrepância entre a figura atual e a } \\
\text { ideal }\end{array}$ & $3,5 \pm 1,9(3,5 ; 1-7)$ & $2,6 \pm 1,8(2 ; 0-7)^{*}$ \\
\hline
\end{tabular}

a : valores expressos como média \pm desvio-padrão (mediana; mínimo - máximo). * $: p \leq 0,05$.

No que diz respeito à escolha da silhueta atual pelas participantes, não houve diferença significativa em relação ao pré e ao pós-tratamento $(\mathrm{z}=-0,9, \mathrm{p}=0,4)$, porém houve uma tendência de diminuição. No pré-tratamento, houve, em média, a escolha pela silhueta 5 , que corresponde a um IMC $>20$ e $\leq 30 \mathrm{~kg} / \mathrm{m}^{2}$, sendo que no pós-tratamento essa média diminuiu para a silhueta 4 aproximadamente (média $=3,9$ ).

No pré-tratamento, seis $(42,9 \%)$ das mulheres superestimaram seu tamanho e no pós-tratamento apenas três $(27,3 \%)$ superestimaram. Já oito $(57,1 \%)$ dessas mulheres não superestimaram seu tamanho corpóreo no pré-tratamento e no pós também oito $(72,7 \%)$ das participantes que não desistiram não superestimaram. Não houve diferença significativa, pelo teste do Qui-quadrado, na proporção de pacientes que superestimavam seu tamanho corporal antes e depois do tratamento $\left[x^{2}=0,65(0,5<\mathrm{P}<0,25)\right]$. 
As pontuações obtidas nas subescalas do questionário de atitude estão descritas na tabela 2.

Tabela 2. Distribuição das variáveis (em média, desvio-padrão, mediana, mínimo e máximo) das subescalas do Body Attitudes Questionnaire-BA0, nos períodos pré e pós-tratamento

\begin{tabular}{l|c|c}
\hline & Pré-tratamentoa & Pós-tratamentoa \\
\hline Atração física & $15 \pm 4,2(5,5 ; 6-21)$ & $15,3 \pm 2,8(16 ; 11-20)$ \\
\hline Depreciação & $25,6 \pm 7,5(26 ; 12-38)$ & $21,3 \pm 7,8(21 ; 11-37)^{*}$ \\
\hline Sentir-se gorda & $57,2 \pm 5,5(57,5 ; 46-65)$ & $48 \pm 9,2(48 ; 30-59)^{*}$ \\
\hline Saliência & $31,4 \pm 4,8(31,5 ; 24-40)$ & $25 \pm 7,1(25 ; 14-35)^{*}$ \\
\hline $\begin{array}{l}\text { Gordura dos membros } \\
\text { inferiores }\end{array}$ & $16,9 \pm 2,4(16 ; 12-20)$ & $14,1 \pm 2,6(14 ; 10-18)^{*}$ \\
\hline Força e aptidão física & $16,9 \pm 4,5(17,5 ; 8-25)$ & $19,1 \pm 3,3(19 ; 14-24)$ \\
\hline
\end{tabular}

a : valores expressos como média \pm desvio-padrão (mediana; mínimo - máximo). * $: p \leq 0,05$.

No presente estudo, foi analisado se as pacientes que abandonaram o tratamento eram mais graves segundo a frequência total de episódios de $\mathrm{BN}$ e purgativos, pois, se isso se afirmasse, os efeitos positivos observados no pós-tratamento poderiam ser em função da desistência dessas pacientes, em vez de ser pelo real efeito do tratamento. Não houve diferença significativa nos episódios purgativos nem bulímicos $(\mathrm{z}=-0,39, \mathrm{p}=0,7 \mathrm{e} \mathrm{z}=-0,62, \mathrm{p}=0,53$ respectivamente), portanto, segundo a análise estatística, quem abandonou o tratamento não foram as participantes mais graves sob o aspecto dos critérios da BN.

\section{Discussão}

O objetivo principal deste trabalho foi acompanhar a evolução dos distúrbios da imagem corporal em pacientes com BN durante tratamento multidisciplinar de 18 semanas. Notou-se que, no pré-tratamento, mais do que a metade $(57,1 \%)$ das participantes não superestimavam seu tamanho corporal e as participantes que desistiram do tratamento não eram as mais graves. Em relação ao pós-tratamento, houve aumento da figura escolhida como ideal, diminuição da discrepância entre as figuras atual e a saudável e diminuição da discrepância entre as figuras atual e a ideal. Assim, constata-se que houve diminuição da insatisfação corporal. Em relação às atitudes corporais, houve diferença significativa nos escores das seguintes subescalas: sentir-se gorda, saliência, gordura nos membros inferiores e depreciação. Além disso, as pacientes que não remitiram dos sintomas bulímicos tinham maior pontuação nas subescalas de depreciação e saliência.

Houve uma diminuição significativa dos sintomas de BN no pós-tratamento. A terapia, a intervenção nutricional, o suporte psiquiátrico, a indicação do uso de medicamentos específicos e a diminuição da restrição ajudam a diminuir a ansiedade, a tristeza e outros sentimentos considerados facilitadores de episódios de compulsão alimentar e de indução de vômitos ${ }^{18,19}$.

Quanto ao IMC médio inicial e final da amostra, não houve diferença significativa $(p=0,6)$, porém houve uma leve tendência a aumentar, sendo que no pré-tratamento o IMC médio foi de 22,7 $\pm 4,1$ e, no pós-tratamento, de $23,0 \pm 4,5$. Tanto no pré quanto no pós-tratamento o IMC médio se encontrava na faixa de eutrofia $(18,5$ $\left.\leq \mathrm{IMC}<25 \mathrm{~kg} / \mathrm{m}^{2}\right)$.

Como apresentado anteriormente, houve aproximadamente $21,4 \%$ de desistência durante o tratamento, e as pacientes que desistiram não eram significativamente mais graves do que as que permaneceram até o final do tratamento em relação aos sintomas de $\mathrm{BN}$, como visto nos resultados. Em estudos prévios $2,20,21$, houve uma similar taxa de abandono do tratamento $(29,4 \%$ e $33 \%$ respectivamente). Segundo os autores, as pacientes que abandonaram tinham mais transtorno de personalidade diagnosticado pelo Personality Diagnostic Questionnaire (PDQ) ${ }^{22}$. Uma das limitações do presente estudo foi justamente não ter aplicado algum instrumento que mensurasse transtorno de personalidade para possível correlação com prognóstico, desistência e/ou remissão de sintomas.
Em relação aos dados correspondentes diretamente com a imagem corporal, pode-se perceber na tabela 1 que não houve diferença significativa da escolha da figura atual entre o pré e o póstratamento. Porém, houve no pré-tratamento, em média, a escolha pela figura 5 , que equivale a um IMC $\geq 25 \mathrm{e}<30 \mathrm{~kg} / \mathrm{m}^{2}$, sendo que no pós-tratamento essa média diminuiu para a figura 4 , equivalente a um IMC $\geq 18,5$ e $<25$ (média $=3,9$ ). Essa diminuição da escolha da figura atual é mais condizente ao IMC médio da amostra, que não oscilou significativamente ao longo do tratamento e era eutrófica $(85,7 \%)$, o que pode representar uma aproximação da percepção das pacientes com seu peso real.

No pré-tratamento, apenas seis $(42,9 \%)$ das 14 mulheres superestimaram seu tamanho. Além disso, não houve diferença significativa, pelo teste do Qui-quadrado, na proporção de pacientes que superestimavam seu tamanho corporal antes e depois do tratamento $\left[x^{2}=0,65(0,5<p<0,25)\right]$. É importante citar que a distorção da imagem corporal é critério diagnóstico para anorexia nervosa (AN). No caso da $\mathrm{BN}$, considera-se como critério a insatisfação com o corpo ${ }^{11}$, porém, mesmo sendo a insatisfação corporal o distúrbio mais presente, ele não é o único. Esse estudo tenta aprofundar mais os demais distúrbios atitudinais presentes em pacientes com $\mathrm{BN}$, visto que a distorção realmente não se mostrou predominante entre as participantes do presente estudo.

Alguns estudos apresentaram resultados diferentes, utilizando outras maneiras para mensurar a superestimação corporal. Sunday et al. ${ }^{23}$ acharam um significativo decréscimo da superestimação corporal em pacientes com BN, seguindo tratamento de internação, assim como Peterson et al. ${ }^{2}$ que, em estudo mais recente, notaram uma grande diferença da redução da superestimação corporal de pacientes com BN em comparação com um grupo controle, incluindo a fase de follow-up (6 meses pós-tratamento).

Em relação ao número da figura escolhida como saudável, houve tendência de diminuição, embora não tenha atingido rigorosamente o critério de significância estatística, entre o pré e o pós-tratamento $(\mathrm{z}=-1,86, \mathrm{p}=0,06)$. A média escolhida foi a figura 3 aproximadamente (pré-tratamento $=2,6 /$ pós-tratamento $=3,3$ ), tanto no pré quanto no pós-tratamento. É importante ressaltar que, no pré-tratamento, a figura mínima escolhida como saudável foi a 1 , já no pós-tratamento a menor figura escolhida como saudável foi a 2, que está mais próxima ao limite inferior de eutrofia.

$\mathrm{O}$ número médio da figura ideal no pré-tratamento foi $1,5 \mathrm{e}$ no pós foi 2,4 , sendo a diferença estatística significante $(z=-2,83$, $\mathrm{p}=0,005)$. A menor figura escolhida no pré foi a 1 e no pós mudou para a 2. Esse resultado é de extrema importância para reconstrução emocional do desejo de ser magra ${ }^{15}$. Uma vez que as participantes têm um desejo mais palpável e realista, pode ser que não haja tanta insatisfação acerca do peso atual, que está muito mais próximo da figura 2 do que a 1 . Mesmo não tendo um número específico para cada figura, pressupõe-se que o IMC em torno de $23 \mathrm{~kg} / \mathrm{m}^{2}$ (que foi a média das participantes no pós-tratamento) esteja mais próximo da figura 2 do que da figura 1 .

A discrepância entre a figura escolhida como atual e saudável representa uma medida de insatisfação corporal mais realista e racional ${ }^{15}$. Esta, por sua vez, obteve uma diferença significativa $(\mathrm{z}=$ $-2,16, \mathrm{p}=0,03)$ no final do tratamento, sendo um aspecto positivo por possivelmente contrabalançar o aspecto mais emocional ${ }^{15}$.

Quanto à discrepância entre a figura atual e a ideal, também houve diferença $(z=-2,06, p=0,04)$ no pré e no pós-tratamento. A discrepância diminuiu da média 3,5 para 2,6, o que representa uma mudança muito positiva e benéfica do ponto de vista da decisão emocional característica dessa variável, pois, na grande maioria das vezes, as pacientes desejam ter um corpo demasiadamente magro que não condiz com sua estrutura genética e, portanto, acabam se frustrando ciclicamente ao desejar ter um corpo que nunca conseguirão ter, o que aumenta a insatisfação corporal e piora, consequentemente, a BN.

Em relação às subscalas do BAQ, houve diferença no quesito depreciação $(z=-2,22, p=0,03)$, que significa um resultado extremamente positivo, pois algumas perguntas dessa subescala questionavam sobre constrangimentos em relação à aparência, na presença de outros indivíduos. 
A saliência pode ser interpretada como a importância que a pessoa denota para seu próprio corpo e forma. Houve uma mudança significativa $(\mathrm{z}=-2,81, \mathrm{p}=0,005)$ da importância que as participantes davam para seus corpos no início e no final do tratamento. Elas passaram a se importar menos, mesmo estando com o IMC levemente maior.

Já a subescala condizente com a força e aptidão física não se apresentou significativa $(z=-1,74, p=0,08)$, porém houve uma leve tendência a aumentar. $\mathrm{O}$ atual padrão de beleza é, além de ser magro, ser musculoso ${ }^{23}$, o que denota um resultado positivo de autoestima, uma vez que as participantes estão tendendo a se achar mais fortes em vez de mais gordas.

De todas as variáveis analisadas entre as pacientes que tiveram e as que não tiveram remissão total de sintomas, as únicas que apresentaram uma diferença estatisticamente significativa foram a depreciação e a saliência. Estas estavam muito aumentadas nas pacientes que não remitiram, podendo-se sugerir a hipótese de que quem dá maior importância para o corpo (saliência) e/ou tende a se depreciar mais não obtém um resultado tão positivo no tratamento.

As variáveis relacionadas à percepção não foram, depois de feitas algumas análises, o foco central do problema de distúrbios da imagem corporal como mostram muitos artigos e sim atitudes específicas em relação a alguns aspectos da imagem corporal, tais como depreciação e saliência, que apontaram diferenças nas participantes que ainda continuaram com algum sintoma.

Após o tratamento de 18 semanas, houve uma alta porcentagem de pacientes com remissão de sintomas, e, depois de feita a comparação com as que desistiram no meio do tratamento, pode-se concluir que as que saíram não eram as mais graves, ressaltando a eficácia do tratamento naquelas que permaneceram. Porém, não foi realizado um acompanhamento para continuar observando os benefícios do tratamento. Em relação à insatisfação da imagem corporal, houve diferença na figura escolhida como ideal, que se aproximou mais da figura média atual. Houve diferença nas discrepâncias entre as figuras escolhidas como atual e saudável e entre atual e ideal, que são, respectivamente, medidas mais racionais e emocionais da insatisfação corporal ${ }^{15}$. Não houve, porém, diferença em relação à superestimação corporal e o tratamento poderia ser refinado nesse aspecto.

Já em relação às atitudes, houve uma diminuição significativa das seguintes variáveis no pós-tratamento: sentir-se gorda; saliência; gordura nos membros inferiores e depreciação.

Esses resultados são extremamente benéficos, pois mostram que o tratamento conseguiu abordar e modificar essas atitudes que são marcantes e muito presentes nas pacientes com TA e podem ser preceptivas de uma cronificação e agravo da doença, se não trabalhadas.

Não se sabe, ao certo, qual dos componentes desse tratamento exerceu mais efeito sobre a imagem corporal. De forma geral, o tratamento oferecido pela unidade provocou alterações positivas nos distúrbios de imagem corporal.

\section{Agradecimentos}

Agradecemos ao Ambulim por facilitar a realização deste projeto. Apoio: Fundação de Amparo à Pesquisa do Estado de São Paulo (Fapesp) (processo - 04/11608-0).

\section{Referências}

1. Skrzypek S, Wehmeier PM, Rremschmidt H. Body image assessment using body size estimation in recent studies on anorexia nervosa. A brief review. Eur Child Adol Psychiatry. 2001;10:215-21.
2. Peterson CB, Wimmer S, Ackard DM, Crosby R, Cavanagh LC, Engbloom S, et al. Changes in body image during cognitive-behavioral treatment in women with Bulimia Nervosa. Elsevier; 2004. p. 139-53.

3. Hraboskt JI, Cash TF, Veale D, Neziroglu F, Soll EA, Garner DM, et al. Multidimensional body image comparisons among patients with eating disorders, body dysmorphic disorder, and clinical controls: a multisite study. Body Image. 2009;6(3):155-63.

4. Slade PD. What is body image? Behav Res Ther. 1994;32:497-502.

5. Muth JL, Cash TF. Body-image attitudes: what difference does gender makes? J Appl Soc Psy. 1997;27:1428-52.

6. Benninghoven D, Jurgens E, Mohr A, Heberlein E, KuzendorfS, Jantschek G. Different changes of body-images in patients with anorexia or bulimia nervosa during inpatient psychosomatic treatment. Eur Eat Disor Rev. 2006;14(2):88-96.

7. Cash TF, Fleming EC. The impact of body image experiences: development of the body image quality of life inventory. Inter Jour Eat Disor. 2002;31:455-60.

8. Ben-Tovim DI, Walker MK. The influence of age and weight on women's body attitudes as measured by the Body Attitudes Questionnaire (BAQ). J Psychosom Res. 1994;38:477-81.

9. Alvarenga MS. Bulimia nervosa: avaliação do padrão e comportamento alimentar. [doutorado]. Universidade de São Paulo (USP): São Paulo; 2001.

10. Cordás TA. Avaliação da eficácia terapêutica de dois modelos de atendimento em bulimia nervosa. [doutorado]. Faculdade de Medicina da Universidade de São Paulo (FMUSP). São Paulo; 1995.

11. American Psychiatric Association (APA). Diagnostic and Statistical Manual of Mental Disorders (DSM-IV-TR). 4th ed. Washington, DC: APA Press; 2000

12. Hamilton M. A rating scale for depression. J Neurol Neurosurg Psychiatry. 1960;23:56-62.

13. Salzano FT, Cordás TA. Tratamento farmacológico de transtornos alimentares. Rev Bras Psiquiatr. 2004;31(4):188-94.

14. Scagliusi FB, Polacow VO, Cordás TA, Coelho D, Alvarenga M, Philippi ST, et al. Validity of the Body Attitudes Questionnaire. Appetite; 2005.

15. Stunkard AJ. A history of binge eating. In: Fairburn CG, Wilson GT, editors. Binge eating: nature, assessment and treatment. New York: Guilford; 1993. p. 15-34.

16. Scagliusi FB, Polacow VO, Cordás TA, Coelho D, Alvarenga M, Philippi ST, et al. Tradução, adaptação e avaliação psicométrica da Escala de Conhecimento Nutricional do National Health Interview Survey Cancer Epidemiology. Rev Nutr Campinas. 2006;19(4):425-36.

17. Bulik CM, Wade TD, Heath AC, Martin NG, Stunkard AJ, et al. Relating body mass index to figural stimuli: population-based normative data for Caucasians. Int J Obs. 2001;25(10):1517-24.

18. Duchesne M, Almeida PEM. Cognitive-behavioural therapy of eating disorders. Rev Bras Psiquiatr 2002;24(3):49-53.

19. Shapiro JR, Berkman ND, Brawley KA, Sedway JA, Lohr KN, Bulik CM. Bulimia nervosa treatment. A systematic review of randomized controlled trials. Int J Eat Disord. 2007;40:321-36.

20. Tanesi PHV, Yazigi L, Fiore MLM, Pitta JCN. Adesão ao tratamento clínico no transtorno de personalidade borderline. Estudos de Psicologia. 2007;12(1):71-8.

21. Fairburn CG, Peveler RC, Jones R, Hope RA, Doll HA. Predictors of 12-mounth outcome in bulimia nervosa and the influence of attitudes to shape and weight. J Consult Clin Psychol. 1993;61:696-8.

22. Johnson JG, Bornstein RF. Utility of the Personality Diagnostic Questionnaire- Revised in a non-clinical population. J Personal Disord. 1992;6(4):450-7.

23. Sunday SR, Einhorn A, Halmi KA. Relashionship of perceived macronutrient and caloric content to affective cognitions about food in eating-disordered, restrained, and unrestrained subjects. Am J Clin Nutr. 1992;55:362-71. 